\title{
Computer Ethics in the Semantic Web Age
}

\author{
Aziz Alotaibi \\ Department of Computer Science \\ 221 University Ave, University of Bridgeport \\ Bridgeport, CT, USA
}

\begin{abstract}
Computer ethics can be defined as a set of moral principles that monitor the use of computers. Similar rules were then required for both programmers and users. Issues that were not anticipated in the past have arisen due to the introduction of newer platforms such as Semantic Web. Both programmers and users are now obliged to consider phenomenon such as informed consent. In this paper, I will explore the ethical problems that arise for professionals and users with the advent of new technologies, especially with privacy concerns and global information.
\end{abstract}

Keyword-Computer ethics; semantic web; privacy concerns; and global information

\section{INTRODUCTION}

In early 1940s, the computer ethics field was founded by MIT professor Norbert Wiener as academic filed[1]. With advancements made in computer science, web technologies provided us with the opportunity to explore a whole new world that we were previously unaware of due to our limited access to resources. However, this knowledge came at a cost[2]. Individuals can no longer be assured that their privacy is maintained on the web. First, people's privacy is being threatened by hackers and computer crime since their information is being controlled by other entities, such as government agencies or other private enterprises.

Nowadays, people have share personal information at a much greater scale than in the past; for instance, their lifestyle, information about their health, their financial status, their political views, their religious affiliation, and gender. Thus, there is greater paranoia in their minds that their information might be accessed by computer hackers. From the hacker's perspective, there should be no restriction to accessing people's information. However, that is not the case and information is being strictly controlled through various means to protect it from being easily shared. Yet, in the new age world, where information our desire to gather information has reached an all-time high, we pay for our quicker, faster access to services by loosening our grip on our privacy and security on the web.

\section{SEMANTIC WEB}

In 1989, Tim Berners-Lee, at CERN (European Organization for Nuclear Research), made a proposal for a system that would enable people to share the information using the hypertext system. And in 1990, he wrote the first document using HTML[3]. As we know WWW is an abbreviation for World Wide Web which known as (web). And he invented the HTML and HTTP protocol. HTML, stands for hypertext markup language, is used to create and structure documents that can be shown as web pages. HTTP, short for the hypertext transfer protocol, is an application protocol that use to request and transmit files over the internet. And he invented the HTML and HTTP protocol. HTML, stands for hypertext markup language, is used to create and structure documents that can be shown as web pages[4]. HTTP, short for the hypertext transfer protocol, is an application protocol that use to request and transmit files over the internet. Semantic web is known as web 3.0 and an extension to the World Wide Web that enables to share the data.

As Tim Berners-Lee states semantic web is the movement from the web of documents to the web of data [5] and from the human centric to the machine-processable.

\section{TABLE I. WEB GENERATION}

\begin{tabular}{|c|c|c|c|}
\hline $\begin{array}{l}\text { Document } \\
\text { web 1.0 }\end{array}$ & Social web 2.0 & $\begin{array}{l}\text { Semantic web } \\
\text { 3.0 }\end{array}$ & $\begin{array}{l}\text { Ubiquitous } \\
\text { web } 4.0\end{array}$ \\
\hline $\begin{array}{c}\text { Document - } \\
\text { centric } \\
\text { HTML, CSS, } \\
\text { Javascript } \\
\text { Search engines } \\
\text { URL }\end{array}$ & $\begin{array}{l}\text { Data-centric } \\
\text { API, web } \\
\text { services, AJAX } \\
\text { Social network } \\
\text { URI }\end{array}$ & $\begin{array}{l}\text { Data-centric } \\
\text { RDF,RDFa, } \\
\text { OWL, SPARQL } \\
\text { Structure data } \\
\text { Linked data }\end{array}$ & $\begin{array}{l}\text { User centric } \\
\text { Invisible web } \\
\text { Smart market } \\
\text { Semantic } \\
\text { mobile services }\end{array}$ \\
\hline
\end{tabular}

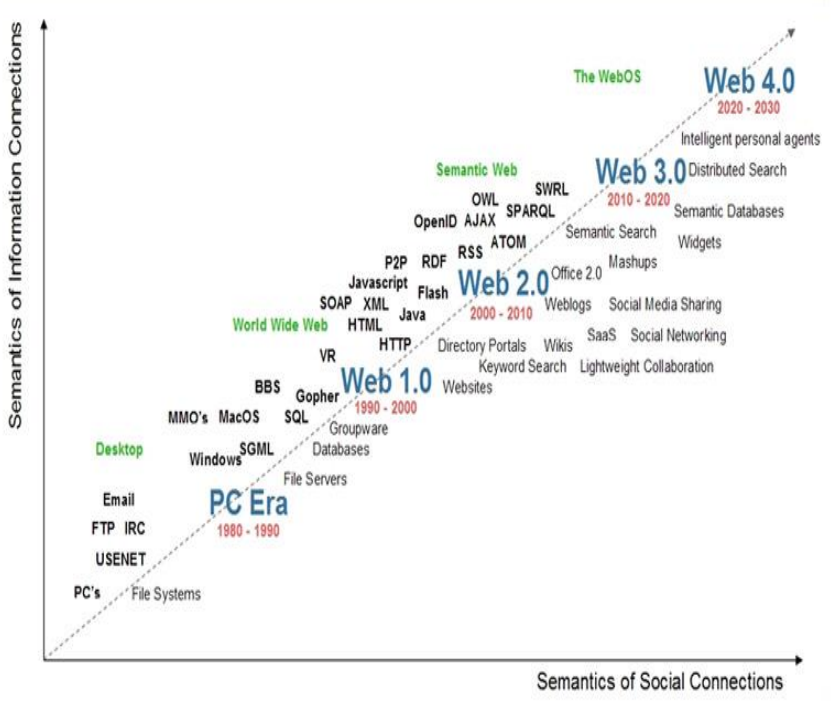

Fig. 1. Source: Radar Networks \& Spivack,2007 www.radarnetworks.com

Since these privacy issues already existed prior to the development of newer technologies such as semantic web, its creators and users may question how the introduction of this new platform will affect an already existing problem, i.e., how 
will the addition and usage of semantic web further intensify privacy control issues. Information gathering and transaction was possible on the web prior to the discovery of semantic web. However, it is essential to understand that the creation of semantic web has added and made the possibility of data sharing significantly easier than in the past.

\section{LINKED DATA}

Linked Data is a structure data that has been published and connected by linking different sources over the web. Before semantic web technology has been used, we use the hypertext links to link documents and browse them on the web. Also we use XML to structure the document. Here is an example of XML:

$<$ Website>

$$
\begin{aligned}
& \text { <creator name="Aziz"> } \\
& \text { <age }>24</ \text { age }> \\
& \text { </creator> } \\
& \text { <uri> } \\
& \text { www.unethicalwebsite.com } \\
& \text { </uri> } \\
& \text { <contributor> } \\
& \text { www.yourwebsite.com } \\
& \text { </contributor> }
\end{aligned}
$$

$</$ Website $>$

It is easy to understand XML file, However, XML cannot provide any relationship between the data. Moreover, we cannot query XML file to provide us with an answer.

Semantic web has many languages, and one of them is RDF which is a graph data format. Semantic web uses RDF (Resource Description framework) technology to link the data. RDF can make a relationship between the data over the World Wide Web[4]. The RDF technology relies on URI (uniform Resource Identifier) to link and describe the data items by providing properties and property values as shown in figure $2[6]$.

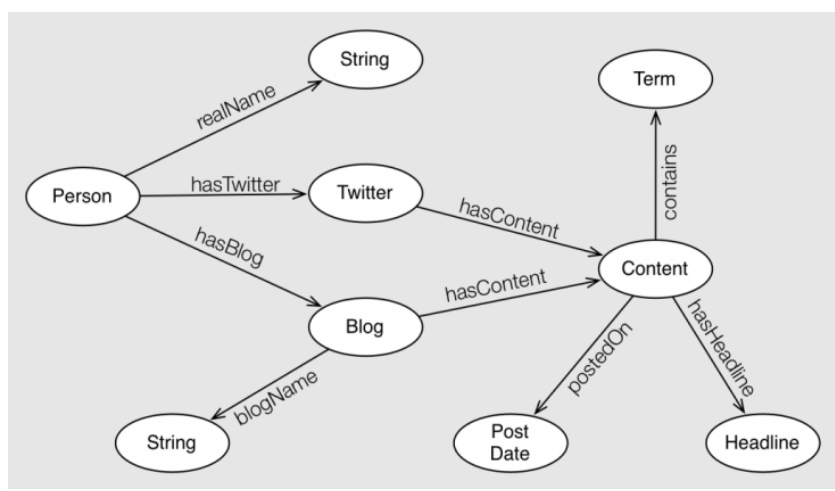

Fig. 2. Linked data

Since these privacy issues already existed prior to the development of newer technologies such as semantic web, its creators and users may question how the introduction of this new platform will affect an already existing problem, i.e., how will the addition and usage of semantic web further intensify privacy control issues. Information gathering and transaction was possible on the web prior to the discovery of semantic web. However, it is essential to understand that the creation of semantic web has added and made the possibility of data sharing significantly easier than in the past.

<rdf: RDF

xmlns:rdf="http://www.w3.org/1999/02/22-rdf-syntax-ns\#"

xmlns:rdfs="http://www.w3.org/2000/01/rdf-schema\#"

xmlns:foaf="http://xmlns.com/foaf/0.1/"

xmlns:dc="http://purl.org/dc/elements/1.1/" >

<rdf:Description rdf:about="http://www.unethicalwebsite.com"> <dc:title> unethical websites </dc:title>

$</$ rdf:Description $>$

In this case, anyone can link you to the website as Contributor. <rdf:Description rdf:about="http://://www.unethicalwebsite.com "> $<\mathrm{dc}$ : Contributor rdf:resource="http:// www.yourwebsite.com"/> $</$ rdf:Description $>$

$</$ rdf:RDF $>$

As explained by Shabajee, semantic web operates on the idea of connecting data and metadata in the easiest possible manner. This is made possible by enabling all data to be viewed through a standard format which was a major obstacle in data sharing in the past. Now, with the option of sharing data through semantic web, it is much easier to violate privacy laws unless stricter measures are put in place.

Another function of semantic web is assuring reusability of the data so as to increase its chances of redistribution which leads to its existence even if the original source is removed from the web at a later stage. Therefore, it is highly likely that even if a user deletes specific content from the original location, several other copies would be easily available as a result of prior distribution of the material. This is an example of the effect of new technology on privacy measures and it calls specific checks in place to maintain privacy on the web. The need to find appropriate measures of regulation is critical, as it is possible to immediately integrate the semantic web version of a statement and represent it in results from queries across the whole web. This poses a definite problem since individuals tend to have a variety of information on the web and prefer to separate their professional information from their personal lives. However, the availability to link data through semantic web would connect results from either aspects of an individual's life and can be misused and cause serious damage to their professional reputation or even make them easy victims of stalkers.

Moreover, people get offended on the internet when criticized for their beliefs and their cultural differences. People hold different beliefs and come from societies; they express their point of view on the internet, which sometimes is considered to be offensive by others. People have strong feelings about a particular issue and in the process of expressing their opinion on the web; they end up unintentionally offending those with a different viewpoint on the subject[1]. Thus, if global interaction has to prosper, we are in dire need of educating our users about social rules and expectations so that they can make informed and ethical decisions. This can be achieved by specific long term education process or through publicity or educational campaigns. Educating users about social media ethics would be a step in the right direction so that they are able to communicate with each other in a fair and respectable manner online. 
This spread of knowledge should not be limited to just users, web designers and developers should be aware of global information ethics. Moreover, existing design and interface options should be further developed to provide consumers with the level of security that they would feel comfortable using. For instance, giving users a general understanding of how their accounts may be linked will prove beneficial in the long run and may prompt them to read the privacy consent with greater caution before granting their approval. As of now, users have inaccurate ideas of the functionality of most web applications and by adopting models that are easy to understand, we can hope for a more informed population that accesses the web in the future and that in turn will help them to make ethical choices in the electronic world as well.

\section{CONCLUSION}

Since the development of computers and the various applications that followed, computer ethics has reached a new found importance due to the emergence of new complications that have accompanied our progress in this field. Traditional web based applications were once considered to be the peak of our grasp over technology. However, human interaction has achieved a new and rapidly evolving form due to the rise of social media and networking which has redefined our understanding of privacy, made us rethink our control over granting consent and altered the means of global information sharing. The recent breakthroughs in technology such as semantic web are indeed a welcome addition; yet they come with the added responsibility on the part of their developers of ensuring privacy and educating both users and themselves about making ethical choices.

\section{REFERENCES}

[1] Bynum, T.W. and S. Rogerson, Computer ethics and professional responsibility. 2003.

[2] Hann, I.H., et al. Analyzing Online Information Privacy Concerns: An Information Processing Theory Approach. in System Sciences, 2007. HICSS 2007. 40th Annual Hawaii International Conference on. 2007.

[3] Guha, R. Toward the Intelligent Web Systems. in Computational Intelligence, Communication Systems and Networks, 2009. CICSYN '09. First International Conference on. 2009.

[4] Bizer, C., The Emerging Web of Linked Data. Intelligent Systems, IEEE, 2009. 24(5): p. 87-92.

[5] http://www.w3.org/2001/sw/, semantic web.

[6] http://www.oraclealchemist.com/news/linked-data-rdf-and-sparql-part$1 /$. 